\title{
Short communication: Genetic lag represents commercial herd genetic merit more accurately than the 4-path selection model
}

\author{
C. D. Dechow*1 and G. W. Rogers $†$ \\ *Department of Animal Science, Pennsylvania State University, University Park 16802 \\ †Geno Global Ltd., N-2326 Hamar, Norway
}

\begin{abstract}
Expectation of genetic merit in commercial dairy herds is routinely estimated using a 4-path genetic selection model that was derived for a closed population, but commercial herds using artificial insemination sires are not closed. The 4-path model also predicts a higher rate of genetic progress in elite herds that provide artificial insemination sires than in commercial herds that use such sires, which counters other theoretical assumptions and observations of realized genetic responses. The aim of this work is to clarify whether genetic merit in commercial herds is more accurately reflected under the assumptions of the 4-path genetic response formula or by a genetic lag formula. We demonstrate by tracing the transmission of genetic merit from parents to offspring that the rate of genetic progress in commercial dairy farms is expected to be the same as that in the genetic nucleus. The lag in genetic merit between the nucleus and commercial farms is a function of sire and dam generation interval, the rate of genetic progress in elite artificial insemination herds, and genetic merit of sires and dams. To predict how strategies such as the use of young versus daughter-proven sires, culling heifers following genomic testing, or selective use of sexed semen will alter genetic merit in commercial herds, genetic merit expectations for commercial herds should be modeled using genetic lag expectations.
\end{abstract}

Key words: genetic progress, genetic lag, dairy cattle

\section{Short Communication}

Expectations for the rate of genetic progress in dairy cattle selection schemes are generally derived assuming the 4-path selection model where genes are transmitted to the next generation though sires of bulls (SB), dams of bulls (DB), sires of cows (SC), and dams of cows

Received July 25, 2017.

Accepted December 28, 2017.

${ }^{1}$ Corresponding author: cdechow@psu.edu
(DC; Rendel and Robertson, 1950). The rate of genetic change (Falconer and Mackay, 1996) is then

$$
\Delta G=\frac{\Sigma i r \sigma_{A}}{\Sigma L},
$$

where the product of selection intensity $(\partial)$, accuracy $(\sigma)$, and the additive genetic standard deviation $\left(\sigma_{\sigma}\right)$ for $\mathrm{SB}, \mathrm{DB}, \mathrm{SC}$, and $\mathrm{DC}$ are summed and divided by the sum of generation intervals $(\sigma)$. This equation has routinely been applied to determine how changes to the structure of a breeding program will alter the rate of genetic progress (Nicholas and Smith, 1983; Schaeffer, 2006; Garcia-Ruiz et al., 2016).

The derivation of the 4-path model assumed a closed population (Rendel and Robertson, 1950). Whereas the AI breeding population could be considered a closed population dispersed across many herds, commercial farms using AI sires from other herds are not closed. Moreover, Bichard (1971) demonstrated that genetic merit in commercial herds will increase at the same rate as genetic merit in an elite breeding population. This could only be true under the 4-path model assumptions if selection parameters were the same between elite herds generating AI sires (henceforth referred to as the nucleus) and commercial dairy herds, which is not realistic.

Bichard (1971) derived expectations for genetic merit in commercial herds that can be distilled into the following formula for calculating genetic lag on commercial dairy farms:

$$
\mathrm{Lag} \cong-\left(L_{\mathrm{SC}}+L_{\mathrm{DC}}\right) \times \Delta G+i_{\mathrm{SC}} r_{\mathrm{SC}} \sigma_{A}+i_{\mathrm{DC}} r_{\mathrm{DC}} \sigma_{A},
$$

where Lag $=$ the difference between commercial herds and the nucleus at any given time, the subscript SC refers to sires of commercial cows and the subscript DC refers to dams of commercial cows.

The aim of this work is to clarify whether genetic merit in commercial herds is more accurately reflected under the assumptions of the 4-path genetic response formula or by the genetic lag formula. 
Table 1. Four-path ${ }^{1}$ model $(\Delta \sigma)$ and genetic lag (Lag) predictions in terms of genetic SD $(\sigma)$ or years assuming an accuracy of $80 \%$ and for the given selection intensities $(\sigma)$ and generation intervals $(\sigma)$ for nucleus and commercial herds that use randomly selected nucleus bulls when young $\left(\mathrm{L}_{\mathrm{SD}} 2\right)$ or old $\left(\mathrm{L}_{\mathrm{SD}} 6\right)$

\begin{tabular}{|c|c|c|c|c|c|c|c|c|}
\hline \multirow[b]{2}{*}{ Herd } & \multicolumn{3}{|c|}{$\sigma$} & \multicolumn{2}{|l|}{$\sigma$} & \multirow[b]{2}{*}{$\Delta \sigma$} & \multicolumn{2}{|c|}{ Lag } \\
\hline & $\mathrm{SB}, \mathrm{DB}$ & $\mathrm{SC}$ & $\mathrm{DC}$ & $\mathrm{SB}, \mathrm{DB}, \mathrm{DC}$ & $\mathrm{SC}$ & & $\sigma$ & Years \\
\hline Nucleus & 1.75 & 1.75 & 1.75 & 2 & 2 & 0.70 & 0.0 & 0 \\
\hline $\mathrm{L}_{\mathrm{SD}} 2$ & 1.75 & 0 & 0 & 2 & 2 & 0.35 & -2.8 & 4 \\
\hline $\mathrm{L}_{\mathrm{SD}} 6$ & 1.75 & 0 & 0 & 2 & 6 & 0.23 & -5.6 & 8 \\
\hline
\end{tabular}

We assume that generation intervals (2 yr), accuracies $(80 \%)$, and selection intensities $(1.75=$ the top $10.1 \%$ selected) are equivalent among the 4 pathways within the nucleus (Table 1). Based on current reliability for genomic prediction and recently observed generation intervals for AI bulls, these numbers are reasonable approximations of what can be achieved in the SB and DB pathways under genomic selection. Garcia-Ruiz et al. (2016) reported that generation intervals for SB and DB were $<2.5 \mathrm{yr}$ in 2015 and still declining. Generation intervals for SC and DC were reported to be 5.2 and $3.5 \mathrm{yr}$, respectively; however, those values represented generation intervals for commercial cows and here we are concerned with generation intervals for the elite genetic nucleus. Generation intervals and accuracy for SC and DC are similar to those for SB and DB in the elite breeding population because genomically tested virgin heifers are routinely flushed to generate both AI sires and the next generation of elite heifers.

The median net merit $\$$ for genomic young Holsteins bulls with semen available in the United States is in the 92nd percentile (NAAB, 2017), which yields a similar selection intensity (1.86) to our assumption of 1.75. Additionally, our assumed selection differential of 1.4 genetic standard deviations $(0.80 \times 1.75)$ is similar to recent estimates for SB and DB pathways. The genetic standard deviation for milk yield is reported to be 610 $\mathrm{kg}$ (VanRaden, 2017), and reported selection differentials for SB (919 kg) and DB (752 kg; Garcia-Ruiz et al., 2016) are equivalent to 1.51 and 1.23 genetic standard deviations, respectively.

In Table 1, we report selection intensities and generation intervals for 2 commercial herd scenarios plus the resultant rates of genetic progress and genetic lag predicted for each scenario. These parameters were selected to easily contrast 4-path model and genetic lag assumptions. Accuracies (80\%) are the same across scenarios for all pathways, and generation intervals for SB, $\mathrm{DB}$, and $\mathrm{DC}$ are set to $2 \mathrm{yr}$. Selection intensity for SC and DC pathways are set to 0 for both commercial herd scenarios to simplify comparisons. In the first commercial herd scenario $\left(\mathbf{L}_{\mathrm{SC}} \mathbf{2}\right)$, the generation interval for the SC pathway is also 2 yr. The SC generation interval was increased to $6 \mathrm{yr}$ for the second scenario $\left(\mathbf{L}_{\mathbf{S C}} \mathbf{6}\right)$.

The expected rate of genetic progress for $\mathrm{L}_{\mathrm{SC}} 2$ is onehalf that of the nucleus because of the 0 selection intensity for $\mathrm{SC}$ and $\mathrm{DC}$ pathways, and is lowest for $\mathrm{L}_{\mathrm{SC}} 6$. The lag between the nucleus and $\mathrm{L}_{\mathrm{SC}} 2$ was -2.8 genetic standards deviations, which is equivalent to $4 \mathrm{yr}$ of genetic progress in the nucleus. The 4-yr lag is equivalent to the sum of the SC and DC generation intervals, as is the 8-yr lag predicted for $\mathrm{L}_{\mathrm{SC}} 6$. The genetic lag in these scenarios is equivalent to the sum of parent generation interval because the selection intensities for SC and DC are 0 , and the lags would be lessened in practice because superior sires would be selected from the nucleus.

In Table 2, we contrast genetic standard deviations of change in $\mathrm{L}_{\mathrm{SC}} 2$ and $\mathrm{L}_{\mathrm{SC}} 6$ by considering the genetic merit transmitted to cows in such herds by their sire and dam. As selection intensities in the SC and DC pathways are set to 0 , sire transmitting ability (TA) is simply half the average breeding value (BV) of the nucleus 2 yr prior for $\mathrm{L}_{\mathrm{SC}} 2$ and 6 yr prior for $\mathrm{L}_{\mathrm{SC}} 6$. For instance, genetic merit for $\mathrm{L}_{\mathrm{SC}} 2$ in yr 3 (0.35) was half the genetic merit of the nucleus in yr 1. Likewise, dam TA is half the average BV of the commercial herd $2 \mathrm{yr}$ prior. Genetic merit in yr 5 for $\mathrm{L}_{\mathrm{SC}} 2$ cows is 1.23 , which is half of the nucleus BV in yr $3(2.10 / 2=1.05)$ plus half of the cow BV from yr $3(0.35 / 2=0.18)$. Nucleus and commercial herds are assumed to begin with a genetic merit of zero in yr 0 , and genetic improvement in the commercial herds does not begin until calves sired by nucleus bulls are born in yr $3\left(\mathrm{~L}_{\mathrm{SC}} 2\right)$ or yr $7\left(\mathrm{~L}_{\mathrm{SC}} 6\right)$.

The rate of genetic progress is the same in $\mathrm{L}_{\mathrm{SC}} 2$ and $\mathrm{L}_{\mathrm{SC}} 6$ and the $\mathrm{BV}$ of $\mathrm{L}_{\mathrm{SC}} 6$ is identical in yr 10 to that of $\mathrm{L}_{\mathrm{SC}} 2$ in yr 6 when considering parent TA. After $25 \mathrm{yr}$ of selection, the system has stabilized and the genetic merit of $\mathrm{L}_{\mathrm{SC}} 2(-2.799$ genetic $\mathrm{SD})$ and $\mathrm{L}_{\mathrm{SC}} 6(-5.598$ genetic SD) lag behind the nucleus by the approximate amount predicted in Table 1 by the genetic lag formula. The genetic lag would be expected to be exact over an infinite number of generations. The results of Table 2 are plotted over a long-term (50 yr) selection horizon along with the predicted rate of genetic progress from 
Table 2. Transmitting abilities (TA) for sires and dams, cow breeding values (BV), and genetic lag after 25 yr of selection for commercial herds that use randomly selected sires when young $\left(\mathrm{L}_{\mathrm{SD}} 2\right)$ or old $\left(\mathrm{L}_{\mathrm{SD}} 6\right)$ from a nucleus whose genetic merit increases by $0.7 \mathrm{SD}$ per year

\begin{tabular}{|c|c|c|c|c|c|c|c|}
\hline Year & Nucleus & \multicolumn{3}{|c|}{$\mathrm{L}_{\mathrm{SD}} 2$} & \multicolumn{3}{|c|}{$\mathrm{L}_{\mathrm{SD}} 6$} \\
\hline 2 & 1.40 & 0.00 & 0.00 & 0.00 & 0.00 & 0.00 & 0.00 \\
\hline 3 & 2.10 & 0.35 & 0.00 & 0.35 & 0.00 & 0.00 & 0.00 \\
\hline 4 & 2.80 & 0.70 & 0.00 & 0.70 & 0.00 & 0.00 & 0.00 \\
\hline 5 & 3.50 & 1.05 & 0.18 & 1.23 & 0.00 & 0.00 & 0.00 \\
\hline 8 & 5.60 & 2.10 & 0.88 & 2.98 & 0.70 & 0.00 & 0.70 \\
\hline 9 & 6.30 & 2.45 & 1.18 & 3.63 & 1.05 & 0.18 & 1.23 \\
\hline 10 & 7.00 & 2.80 & 1.48 & 4.29 & 1.40 & 0.35 & 1.75 \\
\hline 25 & 17.5 & 8.05 & 6.65 & 14.70 & 6.65 & 5.25 & 11.90 \\
\hline Lag & & & & -2.799 & & & -5.598 \\
\hline
\end{tabular}

the 4-path model in Figure 1. Expectations from the 4-path genetic response formula underpredict progress based on consideration of parent TA for herds using AI sires from the genetic nucleus. Additionally, the choice of using 2-yr-old bulls versus 6 -yr-old bulls results in a growing difference over time when considering the 4-path model; however, the choice of sire age for commercial herds becomes less critical when viewed from a genetic lag perspective as the difference remains identical.

Our genetic lag assumptions refer to commercial herds using AI sires from the elite genetic nucleus. In breeding programs that focus on genetic preservation of regional or native breeds, such as Original Braunvieh or Friesian breeding programs that limit Holstein influence, a widening gap between the elite nucleus and commercial herds as depicted by the dotted lines of Figure 1 is possible.

While genetic progress formulas have proven helpful in defining changes to breeding programs that will accelerate genetic gain in a straightforward manner, they may not capture the full complexity of predicting genetic change in a population. Effects of selection on genetic variation are often ignored when making genetic progress projections. A recent simulation demonstrated that the combined influence of the Bulmer effect, inbreeding, and intense selection can reduce genetic variation by nearly half to the variance of Mendelian sampling (Gorjanc et al., 2015). Overestimations of additive genetic variance can substantially inflate

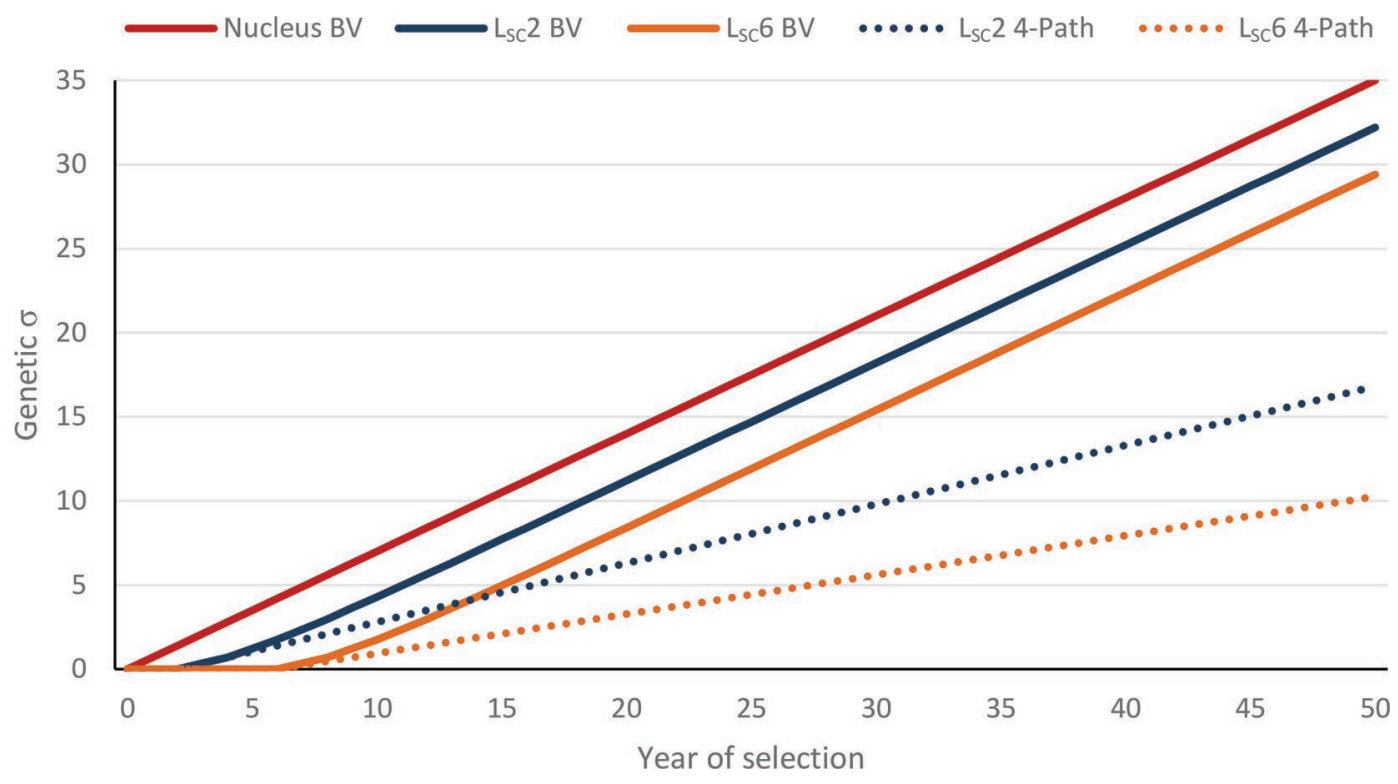

Figure 1. Breeding values (BV) in the genetic nucleus, BV for commercial herds that use randomly selected bulls when young ( $\left.\mathrm{L}_{\mathrm{SC}} 2\right)$ or old $\left(\mathrm{L}_{\mathrm{SC}} 6\right)$, and 4-path model predictions of $\mathrm{BV}$ for $\mathrm{L}_{\mathrm{SC}} 2$ or $\mathrm{L}_{\mathrm{SC}} 6 . \sigma=$ standard deviation. Color version available online. 
accuracy as reliability is typically derived as 1 minus the ratio of prediction error variance to additive genetic variance (Gorjanc et al., 2015). Despite accuracy, selection intensity, and generation interval assumptions that appear reasonable as described above, genetic progress is much lower than the predicted 0.70 genetic standard deviations derived in Table 1 (VanRaden, 2017). This suggests that the effect of variance reduction on genetic progress projections could easily be underestimated and simulation of selection effects may be needed (Gorjanc et al., 2015) to make more accurate projections.

Rates of genetic change for bulls and cows in the US dairy cattle have traditionally been quite similar and in agreement with expectations if we assume a genetic lag between the elite (represented by sires) and commercial (represented by cows) populations. Bull BV can be considered an approximate indicator of genetic merit in the elite AI population which is functionally a dispersed nucleus. The annual rate of increased in bull $\mathrm{BV}$ for protein yield for Holsteins (CDCB, 2017b) from 1990 to 2008 , before the introduction of genomics, was $2.3 \mathrm{~kg}$ per year; cow BV increased at a rate of $2.5 \mathrm{~kg}$ per year during the same period. The genetic lag between bull $\mathrm{BV}$ and cow BV was $10.4 \mathrm{~kg}$ (equivalent to $\sim 4.5 \mathrm{yr}$ ) over the same time period with a range of 8.2 to 10.5 $\mathrm{kg}$. Since the introduction of genomics, genetic merit for protein yield increased at a rate of $3.4 \mathrm{~kg}$ per year from 2009 to 2014 among bulls. This increased rate of gain among bulls is becoming apparent in the cow population, which increased at a rate of $2.8 \mathrm{~kg}$ during the same period, though more time is needed for selection pressures to fully stabilize.

Unfavorable trends in genetic merit in the nucleus will also be transmitted to the commercial cow population, which can be demonstrated by considering the trend for daughter pregnancy rate (DPR; CDCB, 2017a). The genetic trend for DPR was unfavorable until approximately 2000, when selection for productive life and direct selection for DPR (VanRaden et al., 2004) helped to stabilize and eventually reverse the decline. From 1980 to 2000, bull BV for DPR declined by $0.48 \%$ annually and was mirrored by a $0.47 \%$ decline in cow BV. Cow BV remained higher than bull BV by $2.4 \%$ on average (range $=2.0$ to $2.7 \%$ ), demonstrating that unfavorable genetic merit in the dispersed nucleus was transmitted to the commercial population. The genetic lag between bulls and cows was $\sim 4.9$ yr. Since 2009, genetic merit has increased for bulls by $0.60 \%$ who are now transmitting improved DPR to the cow population (0.34\% increase since 2009). Bull BV surpassed cow BV in 2013 and should be expected to remain higher as long as selection favors higher DPR in the elite herds.

The equivalent rates of progress and relatively constant genetic lag between bull BV and cow BV for protein yield and DPR demonstrate that genetic lag is a more appropriate predictor of genetic merit in the commercial population than the 4-path model. If the elite AI population is considered a closed and dispersed population, then the 4-path formula is applicable to the genetic nucleus. The utility of the 4-path model depends on the existence of selection pressure differences in the SC and DC pathways, or between SB and DB pathways in the nucleus, which may be lessened given similar accuracies of heifers and young bulls with genomic testing (VanRaden et al., 2009), and similar generation intervals between bull mothers and sires of sons (Garcia-Ruiz et al., 2016).

The genetic lag formula presented above considers the effect of parent selection on the next generation; it does not consider the effect of genomic testing and culling of potential replacements based on genomic PTA in the current generation. The genetic improvement from such culling can be expressed as $i_{\mathrm{RC}} r_{\mathrm{RC}} \sigma_{A_{\mathrm{RC}}}$, where RC $=$ replacement calves and $\sigma_{A_{\mathrm{RC}}}=$ the genetic standard deviation of replacement calves at the time of selection, which could be reduced relative to population genetic variance if the calves are sired by a small subset of bulls. The lag between the elite nucleus and commercial herds can then be expanded to

$$
\begin{aligned}
& \mathrm{Lag} \cong \\
& -\left(L_{\mathrm{SC}}+L_{\mathrm{DC}}\right) \times \Delta G+i_{\mathrm{SC}} r_{\mathrm{SC}} \sigma_{A *}+i_{\mathrm{DC}} r_{\mathrm{DC}} \sigma_{A^{*}}+i_{\mathrm{RC}} r_{\mathrm{RC}} \sigma_{A_{\mathrm{RC}}},
\end{aligned}
$$

where $\sigma_{\sigma^{*}}=$ additive genetic variance of selection candidates.

In conclusion, genetic merit expectations for commercial herds should be modeled using genetic lag expectations rather than 4-path model expectations. Genetic merit in commercial herds will increase at the same rate as in the elite AI herds regardless of whether herds use young bulls or daughter-proven bulls; however, genetic lag is generally expected to be lower with young bull usage if traits are changing rapidly in the nucleus herds. The genetic lag between commercial herds and the elite AI herds is lowered by reducing the generation interval of sires and dams, increasing EBV accuracy, using bulls of high genetic merit, and selection of superior dams. Although progress and lag formulas provide useful projections of future genetic merit to help guide selection program designs, their precision is affected by change in additive genetic variance due to selection and inbreeding and subsequent effects on reliability.

\section{ACKNOWLEDGMENTS}

This work was supported by the USDA National Institute of Food and Agriculture Federal Appropria- 
tions under project PEN04549 and accession number 1001393.

\section{REFERENCES}

Bichard, M. 1971. Dissemination of genetic improvement through a livestock industry. Anim. Prod. 13:401-411.

CDCB (Council on Dairy Cattle Breeding). 2017a. Trend in Daughter Preg Rate for Holstein or Red \& White. April 2017. Accessed Jul. 13, 2017. https://queries.uscdcb.com/eval/summary/trend.cfm?R Menu=HO.p\#StartBody.

CDCB (Council on Dairy Cattle Breeding). 2017b. Trend in Protein BV for Holstein or Red \& White. April 2017. Accessed Jul. 13, 2017. https://queries.uscdcb.com/eval/summary/trend.cfm?R Menu=HO.d\#StartBody.

Falconer, D. S., and T. F. C. Mackay. 1996. Introduction to Quantitative Genetics. 4th ed. Longman Scientific and Technical, New York, NY

Garcia-Ruiz, A., J. B. Cole, P. M. VanRaden, G. R. Wiggans, F. J. Ruiz-Lopez, and C. P. Van Tassell. 2016. Changes in genetic selection differentials and generation intervals in US Holstein dairy cattle as a result of genomic selection. Proc. Natl. Acad. Sci. USA 113:E3995-E4004.
Gorjanc, G., P. Bijma, and J. M. Hickey. 2015. Reliability of pedigreebased and genomic evaluations in selected populations. Genet. Sel. Evol. 47:65.

NAAB (National Association of Animal Breeders). 2017. Genomic bull database. Accessed Dec. 15, 2017. https://www.naab-css.org/ genomic-bull-database.

Nicholas, F. W., and C. Smith. 1983. Increased rates of genetic change in dairy cattle by embryo transfer and splitting. Anim. Sci. 36:341353.

Rendel, J. M., and A. Robertson. 1950. Estimation of genetic gain in milk yield by selection in a closed herd of dairy cattle. J. Genet. 50:1-8

Schaeffer, L. R. 2006. Strategy for applying genome-wide selection in dairy cattle. J. Anim. Breed. Genet. 123:218-223.

VanRaden, P. M. 2017. Net merit as a measure of lifetime profit: 2017 revision. AIP RESEARCH REPORT NM\$6. February 2, 2017.

VanRaden, P. M., A. H. Sanders, M. E. Tooker, R. H. Miller, H. D. Norman, M. T. Kuhn, and G. R. Wiggans. 2004. Development of a national genetic evaluation for cow fertility. J. Dairy Sci. 87:2285-2292.

VanRaden, P. M., C. P. Van Tassell, G. R. Wiggans, T. S. Sonstegard, R. D. Schnabel, J. F. Taylor, and F. S. Schenkel. 2009. Invited review: Reliability of genomic predictions for North American Holstein bulls. J. Dairy Sci. 92:16-24 